\title{
Fe II Emission in the NLS1 galaxy I Zw 1: Results from NIR Spectroscopy
}

\author{
Alberto Rodríguez-Ardila ${ }^{1}$ and Aurea Garcia Rissmann ${ }^{2}$ \\ ${ }^{1}$ Laboratório Nacioal de Astrofísica/MCT, Brazil \\ Email: aardila@lna.br \\ ${ }^{2}$ Gemini Observatory, Chile \\ Email: arissmann@gemini.edu
}

Keywords. galaxies: Seyfert, infrared: galaxies, techniques: spectroscopic

Fe II comprises up to one third of the line emission in AGNs. For that reason it is an important coolant that needs to be taken into account to fully understand the energetics of the broad line region (BLR). As such, $\mathrm{Fe}^{+}$is a potential diagnostic of gas density, column density, turbulence, temperature and continuum shape. However, understanding Fe II is complicated because of the energy level configuration and different mechanisms that may contribute to the line emission. Sophisticated models need to be employed to fully interpret the observed spectra. Here, nearinfrared (NIR) spectroscopy is used to study the excitation mechanisms of the Fe II. We also derived a NIR semi-empirical template based on the I Zw 1 spectrum that can be fitted to other objects to study this emission.

In this work, we used the theoretical Fe II templates of Sigut \& Pradhan (2003), which includes the effects of Ly $\alpha$ fluorescence, and applied them to observations of I Zw 1 and other Seyfert 1 galaxies with prominent Fe II emission in the optical and NIR region. The main goal is to determine if the secondary cascade lines located near $9200 \AA$, emitted after the capture of a Ly $\alpha$ photon by the $\mathrm{Fe}^{+}$ion, are present in the data and if their relative strengths are compatible with model predictions. Other Fe II NIR lines that are also predicted to be prominent if the fluoresence process is present, notably the $1 \mu \mathrm{m}$ lines (Rodríguez-Ardila et al. 2002), are also expected to be detected.

The Fe II template was first convolved with a Gaussian of $1250 \mathrm{~km} \mathrm{~s}^{-1}$ to match the width of the broad components of the permitted lines of $\mathrm{H}$ I in I Zw 1 . It was then adjusted to the residual Fe II emission of this source, obtained after subtracting the contribution of the continuum and other emission lines. This template succesfully reproduced the observed iron lines, confirming that Ly $\alpha$ fluorescence is a process that indeed needs to be fully taken into account to resolve the long-standing Fe II problem (Wills, Netzer, \& Wills 1985). We also applied this same process to other objects (NLS1 and classical Seyfert 1 galaxies). In most cases, the Fe II template could reproduce the observations, notably the lines located at $9200 \AA, 1 \mu \mathrm{m}, 1.050 \mu \mathrm{m}$, and $1.112 \mu \mathrm{m}$.

Finally, we studied if the NIR emission, measured from the Fe II template and characterized by the $1 \mu \mathrm{m}$ Fe II lines, correlates with the optical emission as measured from the Fe II $\lambda 4570$ bump (data taken from the literature). Our results show that a positive correlation is observed between optical and NIR Fe II, suggesting a common origin of both emissions. In particular, the levels that originate the Fe II lines should be populated mostly by decay of the fluorescence NIR lines.

\section{References}

Rodríguez-Ardila, A., Viegas, S. M., Pastoriza, M. G., \& Prato, L. 2002, ApJ, 565, 140

Sigut, T. A. A. \& Pradhan, A. K. 2003, ApJS, 145, 15

Wills, B. J., Netzer, H., \& Wills, D. 1985, ApJ, 288, 94 